\title{
ECG Reduction for Wearable Sensor
}

\author{
Ragheed Allami, Andrew Stranieri, Venki Balasubramanian \\ Federation University \\ Victoria, Australia \\ ragheed.dawood@yahoo.com
}

\author{
Herbert F. Jelinek \\ Charles Sturt University \\ NSW, Australia \\ HJelinek@csu.edu.au
}

\begin{abstract}
The transmission, storage and analysis of electrocardiogram (ECG) data in real-time is essential for remote patient monitoring with wearable ECG devices and mobile ECG contexts. However, this remains a challenge to achieve within the processing power and the storage capacity of mobile devices. ECG reduction algorithms have an important role to play in reducing the processing requirements for mobile devices, however many existing ECG reduction and compression algorithms are computationally expensive to execute in mobile devices and have not been designed for realtime computation and incremental data arrival. In this paper, we describe a computationally naïve, yet effective, algorithm that achieves high ECG reduction rates while maintaining key diagnostic features including PR, QRS, ST, QT and RR intervals. While reduction does not enable ECG waves to be reproduced, the ability to transmit key indicators (diagnostic features) using minimal computational resources, is particularly useful in mobile health contexts involving power constrained sensors and devices. Results of the proposed reduction algorithm indicate that the proposed algorithm outperforms other ECG reduction algorithms at a reduction/compression ratio $(C R)$ of $5: 1$. If power or processing capacity is low, the algorithm can readily switch to a compression ratio of up to 10:1 while still maintaining an error rate below $10 \%$.
\end{abstract}

Keywords-ECG intervals; compression ratio; data reduction; real-time; energy consumption

\section{INTRODUCTION}

Advances in mobile devices and wearable electrocardiograph (ECG) sensor technology has changed the way ECG signals are collected, stored and processed [1] [2]. This can lead to reductions in the use of Holter devices in favor of real time, continuous monitoring [3]. ECG signals from wearable sensors processed on a battery-driven devices require algorithms to execute rapidly in real-time, despite limitations in processor capability and memory [4].

ECG sensors generate large streams of data that easily exhaust mobile device storage. For instance, at a typical sampling rate between 100 and $500 \mathrm{~Hz}$, where each data point may have 8,12 or 16-bit resolution results in a dataset in the order of $480 \mathrm{Kbits}$ per minute [5]. Some applications are required to process and store ECG recordings for one or more days [4].

The large datasets and limited processing capacity of devices near the sensors results in the need to consider the near, or real-time reduction of large ECG data sets for critical operations in ambulatory recording systems, ECG signal storage systems and for ECG signal transmission over wireless networks [5][6]. However, any reduction in ECG signals should not impede clinicians' diagnostic assessments [7]. Most existing ECG data reduction algorithms are designed to reduce information size while maintaining significant information, so that the ECG wave can be reconstructed from the reduced form as intact as possible [8]. Critically, any ECG data reduction algorithm should preserve the essential features (diagnostic features) of the ECG signal (P, Q, R, S, and T) waves as these peaks and troughs as well as the calculated intervals (i.e. QT interval) are used for the clinical interpretation of the ECG [9] [10].

Existing compression algorithms that achieve considerable compression by applying computationally resource intensive transformations are difficult to deploy on mobile devices, which are constrained by power and memory limitations. In contrast, many reduction algorithms cannot be reversed to re-create the original signal but can reproduce only the important features (diagnostic features) of the signal. Further, many existing reduction algorithms require a block of ECG data and cannot reduce a signal incrementally as it arrives from wearable devices so that the need to store the entire stream can be obviated.

In this paper, we introduce a simple, yet effective, ECG data reduction algorithm where the signal cannot be reconstructed intact but nevertheless has the following advantages:

- The compression/reduction ratio is higher than many existing real-time reduction methods.

- Most ECG diagnostic features, intervals and ECG waves (P, QRS, T) can be detected in the reduced data.

- The algorithm can be applied on programmable ECG mobile sensors such as Shimmer3 ECG sensor and increased battery life.

- Reduction processing is computationally less expensive than current ECG data reduction methods because the algorithm is simple and can execute every few milliseconds.

In the following section, we describe existing real-time reduction algorithms. The algorithms proposed here are presented in Section III before reporting experimental results with standard datasets in Section IV.

\section{ECG DATA REDUCTION METHODS}

Three types of ECG compression/reduction algorithms can be categorized: direct methods (reduction methods), 
transform methods and parameter methods. Direct methods reduce the ECG signal without any preprocessing. They directly detect and eliminate the redundant points from plain signal and provide minimum distortion [11]. This makes direct methods more suited for remote patient monitoring than those in the transformation and parameter methods [12]. Although other methods achieve higher compression ratios than direct methods, they consume more time, power and computational resources to be suitable for deployment with wearable sensors and mobile devices [6][13].

Reduction of ECG signal does not require retaining all data points; rather, only some features of the ECG data are essential ( $\mathrm{P}, \mathrm{Q}, \mathrm{R}, \mathrm{S}, \mathrm{T})$, and relevant intervals. The subtlety of the ECG signal may be ignored in favor of higher compression ratios [11][13].

Most compression and reduction algorithms are evaluated using four fundamental measures; signal reconstruction error, compression ratio, compression performance and root mean square error. The compression ratio (CR) describes the effectiveness of an ECG compression method. CR represents the ratio between the plain ECG signal data size and compressed ECG signal data size [14]. The Percentage Mean Square Difference (PRD) is a computation of error due to signal infidelity. This measures the distortion between the plain and the reconstructed signal [15]. Root Mean Square Error (RMSE) is calculated from actual and expected measurements [16].

Real-time ECG data reduction algorithms known as "lossy" methods are those where the reduced ECG signal usually cannot be reconstructed or recovered exactly. Algorithms in this category include Turning Point (TP), Amplitude Zone Time Epoch Coding (AZTEC), Coordinate Reduction Time Encoding System (CORTES), and Fan/SAPA (Scan Along polygonal Approximation) [9].

Cox et al. [17] introduced the AZTEC algorithm that changes a plain ECG signal into slopes and horizontal lines. The amplitude and length are saved while the horizontal lines use a Zero-Order Interpolator method to reduce the data. Information saved from a slope is the length of the slope and its final amplitude. Although the AZTEC method realizes a high CR of about 10:1 (500 Hz sampled ECG with 12 bits resolution), it provides imprecise reconstruction of ECG waves particularly, T and P waves with a PRD of $5.3 \%$ and requires preprocessing to reduce an ECG signal.

The Turning Point method, proposed by Mueller [18] reduces the sampling frequency of an ECG wave from 200 $\mathrm{Hz}$ to $100 \mathrm{~Hz}$ and preserves the peak of the large amplitude QRS waves. The Turning Point method operates by substituting each of three successive values with the two that best represent the substituted value. The second of the two stored values is used for the computation of the next two values. Turning Point maintains the essential aspects of the ECG data and supplies a CR of 2:1. However, relevant clinical information is lost if a minimum or a maximum value is lost. Therefore, if one of the two values in the pair is a local minimum or a local maximum then it is the other value in the pair that is eliminated [19]. In fact, the proposed method advanced in this article is close to the Turning Point method but it provides higher CR and overcomes the local minimum and local maximum problem that may lead to missed diagnostic features.

The high precision of the Turning Point reduction technique and the superior CR of the AZTEC technique were merged into the CORTES method. Abenstein \& Tompkins [20] demonstrated the implementation analysis of the CORTES, AZTEC, and Turning Point methods for ECG's sampled at $200 \mathrm{~Hz}$ with PRD's 7, 28, and 5, and CRs of 4.8:1, 10:1, and 2:1 respectively.

Fan and SAPA methods represent an ECG data reduction algorithm which provides a $\mathrm{CR}$ of around 3:1 and PRD of nearly 4 (250 Hz sampled ECG). These two methods depend on a First-order Interpolation with two Degrees of Freedom (FOI-2DF) algorithm. The Fan algorithm achieves the FOI-2DF without saving all the raw data samples between the last transmitted and the present sample [21].

The real-time ECG data compression algorithms that can execute on wearable, programmable ECG sensor devices are explained next.

A real-time ECG data compression algorithm, +SLOPE, was introduced by Tai [22]. This algorithm processes some neighboring patterns as a vector and expands the vector if the next pattern falls in a fan spanned by the vector and threshold angle; else, it is a restricted linear segment. By this approach, +SLOPE constantly restricts linear segments of different length. The + SLOPE algorithm reproduces ECG signal however the approach can lead to QRS misdetection and a CR of 4.8 and PRD of 7. The +SLOPE algorithm was tested on MIT-BIH Arrhythmia database.

A compression engine was introduced by Fang et al. [23] for portable real-time ECG data monitoring to transfer ECG signal wirelessly and analyze heart rate variability in realtime. The compression engine achieves a compression ratio of approximately 2.5 by classifying every ECG sample based on prior samples with the Golomb-Rice k-parameter algorithm. The compression algorithm was evaluated on the MIT-BIH Arrhythmia database. The ECG signals were resampled to $256 \mathrm{~Hz}$ before being tested and achieved a compression ratio of approximately 2.5 .

All the methods above provide CR less than the proposed method (except the AZTEC method produces a CR of 10:1 but it does not maintain all relevant clinical data. It typically misses $\mathrm{P}$ and $\mathrm{T}$ waves. The proposed method can provide $\mathrm{CR}$ of about 10:1 and miss P, T waves as well). Also, they require more processing time when they execute on a wearable ECG sensor. Therefore, they consume more energy than the proposed method advanced in this study.

Most recent real-time ECG compression algorithms such as [5] [7] [24] based on transformation techniques require preprocessing and high computational resources [6] [13]. Thus, they are not suited for real-time ECG reduction on wearable sensors and mobile devices because these devices have limitations of CPU execution time, memory capacity and energy consumption.

\section{PROPOSED ECG REDUCTION ALGORITHM}

In this study we used Shimmer3 ECG wearable sensor as a reference to measure energy consumption in milli Joules. The Shimmer3 motherboard consists of low-power Texas 
Instrument 16-bit MSP430F5437A microcontroller, 2.7 Volts, a low-power CC2420 IEEE 802.15.4 compliant radio, and has a Bluetooth module. In addition, Shimmer3 has a $450 \mathrm{mAh}$ lithum-ion rechargeable battery that can work up to 16 hours. The MSP430 microcontroller runs at $8 \mathrm{MHz}$ and has $16 \mathrm{~KB}$ of RAM, $256 \mathrm{~KB}$ of Flash. Also, it has a fast hardware multiplier, but does not have a floating-point unit [25].

The direct "lossy" ECG data reduction algorithm proposed here implements real-time direct reduction. The algorithm reads and stores a window of $n$ consecutive raw ECG measurements at a time. As the window increases in width the error rate in correctly identifying the P, Q, R, S and $T$ points diminishes. A window size of 5 has been determined empirically to produce the optimal tradeoff between compression ratio, accuracy and computational resources. The window size can be varied to achieve different trade-offs depending on the context. The algorithm takes a stream of millivolt readings as input and operates in the same way regardless of the sampling rate. The algorithm, described in Table I operates as follows on a window size of 5.

The algorithm reads 5 consecutive ECG readings. The maximum point of the five samples is identified; if this maximum is positive, it is retained in the reduced set and the other 4 points are discarded. This ensures the $\mathrm{P}, \mathrm{R}$ and $\mathrm{T}$ peaks are not discarded. If the maximum point in the window of 5 is not positive, the minimum is identified and retained and the other 4 are discarded. This ensures the $\mathrm{Q}$ and $\mathrm{S}$ troughs are not missed.

\section{TABLE I. ECG REDUCTION ALGORITHM CODING SCHEMA}

\begin{tabular}{|l|}
\hline ECG Reduction Algorithm \\
\hline Input: Plain ECG signal in $+/$ - millivolts \\
Output: Reduced ECG signal $+/$ - millivolts \\
\\
Step1: window size $(\mathrm{n})=5$ (optimal) \\
Step2: While ECG $\neq$ N Do \\
Step3: Read $n$ \\
Step4: Max $=$ Maximum sample in $n$ \\
Step5: Min $=$ Minimum sample in $n$ \\
Step6: If Max $>=0$ Then store Max \\
Else store Min \\
Step7: Go to step2 \\
\hline
\end{tabular}

The claim advanced in this article is that the algorithm is particularly well suited for the reduction of ECG captured from wearable sensors on mobile patients in real-time and can apply on programmable ECG sensors.

\section{RESULTS}

The proposed algorithms were evaluated using the MITBIH Arrhythmia database [26]. The MIT-BIH database is commonly used in ECG signal analyzing because it includes different patterns of ECG signal and contains 48 ECG for 30 minutes record from different patients. Furthermore, it has two channel ambulatory ECG recordings in digital format with an 11 bits ADC and $360 \mathrm{~Hz}$ sampling rate. We applied the algorithm on all 48 ECG signal records [26].

\section{A. ECG Reduction Results}

Fig. 1 depicts part of the raw ECG from Record No. 100. The red dots represent ECG samples in the raw ECG data. This record represents a normal ECG. Fig. 2 also illustrates a segment of Record 100 but where only some of the samples have been selected for storage by the algorithm described above using a window of size 5. Fig. 2 illustrates that each peak and trough has been correctly identified. For record 100, the algorithm maintains most ECG data and reduces the redundancy. It provides a compression ratio of 5:1 and zero PRD. In addition to the reduction results and accuracy, the algorithm has low complexity and can be applied equally to normal and abnormal ECG signals. Furthermore, it can be used with long and short term ECG signal and data stream.

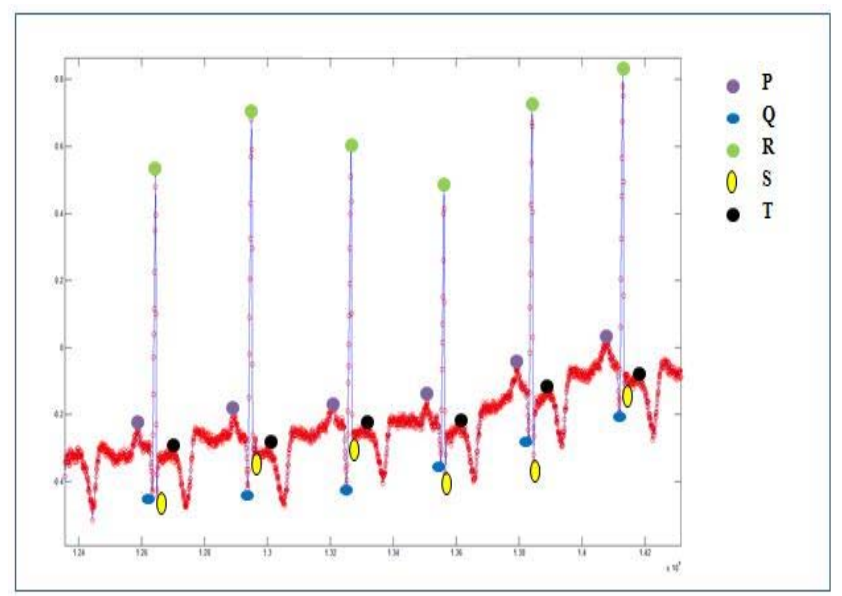

Figure 1. Segment of ECG for Record No. 100 with raw samples.

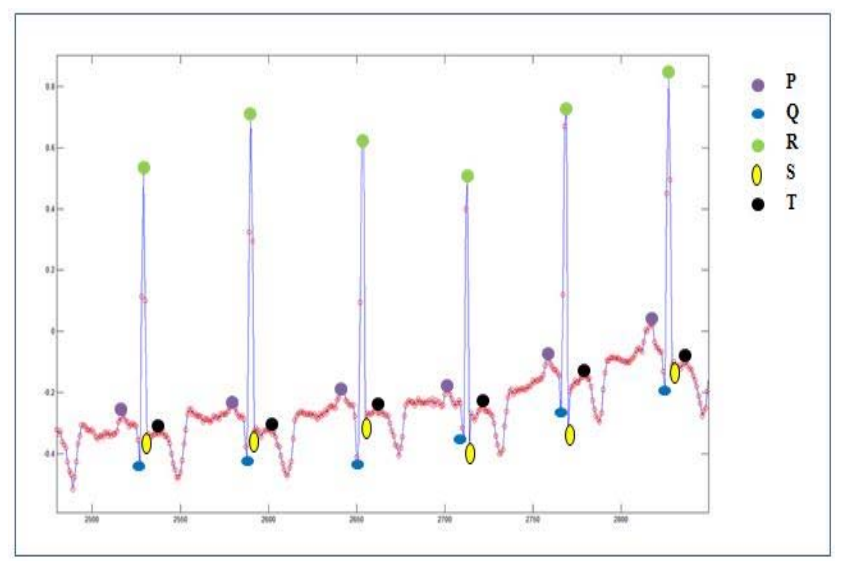

Figure 2. Segment of ECG for Record No. 100 with reduced samples illustrates all ECG peakes and troughes are maintanted after reduction process.

Table II illustrates the proposed algorithm achieves a very low PRD \% at a compression ratio of 5:1 when executed with a window size of 5 . This compares favorably with many other algorithms as depicted in Table II. 
TABLE II. COMPARISON OF ECG DATA REDUCTION ALGORITHMS

\begin{tabular}{|l|c|c|c|}
\hline \multicolumn{1}{|c|}{ Methods } & CR & $\begin{array}{c}\text { Sampling Rate Hz } \\
\text { (No. of Bits) }\end{array}$ & PRD\% \\
\hline TP & $2: 1$ & 200,12 & 5.3 \\
\hline AZTEC & $10: 1$ & 500,12 & 28 \\
\hline CORTES & $4.8: 1$ & 200,12 & 7 \\
\hline FAN/SAPA & $3: 1$ & $250,-$ & 4 \\
\hline SLOPE & $4.8: 1$ & $250,-$ & 7 \\
\hline Fang et al. & $2.5: 1$ & $256,-$ & - \\
\hline Our method & $5: 1$ & Any & 1 \\
\hline
\end{tabular}

The PRD was very low because every point selected was one of the raw ECG points. The compression ratio, accuracy is comparable with the algorithms mentioned in Table II. However, some data is lost with this reduction algorithm in that all points in the window are discarded except for the minimum or maximum. Whether these points are critical for the generation of important ECG indicators is discussed in the next section and illustrates that for ECG features generated from the reduced data, the results are very similar to those generated from the raw data.

\section{B. Generation Features from Reduced ECG Data}

Many methods employed P,QRS,T information to measure the distortion between raw and compresed or reduced ECG signal. For example, Zigel et al. [10] introduced an algorithm to assess the distortion for ECG signal. This algorithm is based on measure $\mathrm{P}, \mathrm{QRS}, \mathrm{T}$ diagnostic features include PR interval, ST segment and RR interval of the raw ECG signal and the compressed ECG signal. Feedback from cardiologists was positive than with traditional distortion measures. Also, the link between diagnostic distortion and PRD metric was categorized as: if the PRD from 0 to $2 \%$ then signal quality is very good, if the PRD from 2 to $9 \%$ then signal quality is very good or good and if the PRD from $>=9 \%$ then signal quality is not possible to determine.

For the evaluation of the proposed method we used $\mathrm{P}, \mathrm{QRS}, \mathrm{T}$ diagnostic features to measure the quality of the reduced signal. Further, we calculated $\mathrm{PRD}$ and $\mathrm{CR}$ parameters that are most commonly used to quantify the reduction effectiveness and accuracy while analyzing the diagnostic quality of the reduced ECG data. CR and PRD are given as below:

$$
\begin{aligned}
& \mathrm{CR}=(\mathrm{X}-\mathrm{Y}) \times 100 \\
& \mathrm{PRD}=\|\mathrm{X}-\mathrm{Y}\|_{2} /\|\mathrm{X}\|_{2} \times 100
\end{aligned}
$$

Where $\mathrm{X}$ is the plain signal and $\mathrm{Y}$ is the reduced signal.

We computed the P,QRS,T dignostice features for the raw data as following:

$$
\mathrm{T}=|\mathrm{i}-\mathrm{N}| \times 1 / \text { frequency }
$$

Where $\mathrm{T}$ represents any interval or segment such as PR interval.

Where $\mathrm{i}$ refers to start intervel and $\mathrm{N}$ referes to end interval.

We reconstructed the dignostic features from the reduced data using the below equetion:

$$
\check{\mathrm{T}}=|(\mathrm{i} \times \mathrm{WS})-(\mathrm{N} \times \mathrm{WS})| \times 1 / \text { frequency }
$$

Where $\check{T}$ represents any interval or segment such as ST segment and WS represents window size.

Most important ECG features that clinics and many computerized algorithms apply to diagnose cardiovascular diseases were generated from the reduced ECG signal. Raw ECG signals were examined with 2, 3, 4, 5, 6, 7, 8, 9 and 10 samples. We utilized the WFDB software that is available publicly to extract ECG features (diagnostic features) from raw and reduced ECG signals [27]. We conclude that most intervals including the QT interval must be accurately detected from reduced ECG signal with a window size of 5 . The RR intervals data detected from the reduced signals have $98 \%$ similarity to the RR data detected from the raw ECG signal up to a window size start from 2 to 10 . Thus, timedomain, frequency-domain, and nonlinear heart rate

\begin{tabular}{|c|c|c|c|c|c|c|c|c|}
\hline Window size & CR & $\begin{array}{c}\text { PRD\% } \\
\text { PR } \\
\text { interval } \\
\end{array}$ & $\begin{array}{c}\text { PRD\% } \\
\text { PR } \\
\text { segment }\end{array}$ & $\begin{array}{c}\text { PRD\% } \\
\text { QRS } \\
\text { interval }\end{array}$ & $\begin{array}{c}\text { PRD\% } \\
\text { ST } \\
\text { segment } \\
\end{array}$ & $\begin{array}{c}\text { PRD\% } \\
\text { ST } \\
\text { interval }\end{array}$ & $\begin{array}{c}\text { PRD\% } \\
\text { QT } \\
\text { interval }\end{array}$ & $\begin{array}{c}\text { PRD\% } \\
\text { RR } \\
\text { interval }\end{array}$ \\
\hline 2 samples & $2: 1$ & 99.9 & 99.9 & 99.9 & 99.9 & 99.9 & 99.9 & 99.9 \\
\hline 3 samples & $3: 1$ & 99.9 & 99.9 & 99.9 & 99.9 & 99.9 & 99.9 & 99.9 \\
\hline 4 samples & $4: 1$ & 99.9 & 99.7 & 99.9 & 99.3 & 99.9 & 99.9 & 99.9 \\
\hline 5 samples & $5: 1$ & 99.2 & 99.6 & 99.2 & 99.1 & 99.2 & 99.5 & 99.4 \\
\hline 6 samples & $6: 1$ & 85.4 & 69.5 & 98.9 & 72.7 & 78.9 & 93.5 & 99.2 \\
\hline 7 samples & $7: 1$ & 71.3 & N/A & 98.3 & N/A & N/A & 83.6 & 98.8 \\
\hline 8 samples & $8: 1$ & N/A & N/A & 98.6 & N/A & N/A & 74.9 & 98.8 \\
\hline 9 samples & $9: 1$ & N/A & N/A & 98.4 & N/A & N/A & 55.9 & 98.6 \\
\hline 10 samples & $10: 1$ & N/A & N/A & 97.9 & N/A & N/A & 55.2 & 98.3 \\
\hline
\end{tabular}
variablity (HRV) analysis results are almost identical to the original ECG signal results.

TABLE III. WINDOW SIZE VERSUS REDUCTION ACCURACY/EFFECTIVENESS

Note: N/A represents failure to detect an interval.

Clearly, there is a link between window size and diagnostic features distortion and detection. When window size set up to $2,3,4$ or 5 the quality of the reduced signal is high and the diagnostic features can be detected. When the 
window size increases to 6 the quality of the reduced signal is good and the diagnostic features can be detected. The quality of the reduced signal decreases when the window size increases above 8,9 or 10 . The $P$ and $T$ waves cannot be detected because of the reduced signal distortion resulting in a failure to detect ST and PR intervals.

\section{Energy consumption for wearable ECG sensor}

The energy consumption has been estimated for the runtime reduction methods by analyzing the number of operations implemented by the micro-controller unit i.e., the number of multiplications, summations, divisions and logical comparisons. These operations have been converted to number of cycles.

$$
\begin{gathered}
\text { Power }=\text { Volts } * \text { Amp } \\
\text { Energy consumption }=\text { Power } * \text { Runtime }
\end{gathered}
$$

TABLE IV demonstrates that the proposed algorithm consumes a very low memory and energy when implement with a window size of 5 .

TABLE IV. COMPARISON OF ECG DATA REDUCTION ALGORITHMS FOR ENERGY CONSUMPTION

\begin{tabular}{|l|c|c|c|}
\hline Methods & $\begin{array}{c}\text { Run Time } \\
\text { ms }\end{array}$ & $\begin{array}{c}\text { Memory } \\
\text { Consumption } \\
\text { KB }\end{array}$ & $\begin{array}{c}\text { Energy } \\
\text { Consumption } \\
\text { mJoule }\end{array}$ \\
\hline TP & 73 & 0.36 & 1.09 \\
\hline AZTEC & 150 & 1.23 & 2.25 \\
\hline CORTES & 248 & 0.97 & 3.72 \\
\hline FAN/SAPA & 100 & 0.75 & 1.5 \\
\hline SLOPE & 267 & 5 & 4 \\
\hline Fang et al. & 235 & 1.40 & 3.52 \\
\hline Our method & 75 & 0.39 & 1.1 \\
\hline
\end{tabular}

The memory and energy consumption are comparable with the algorithms listed in Table IV and is particularly good given that the algorithm executes in real-time and increases battery life using high $\mathrm{CR}$, minimal CPU, storage requirements and power consumption.

\section{CONCLUSION}

The recording of ECG data generates a large amount of data that increases with different sampling rates and time. Nowadays, mobile devices are being widely used in healthcare applications to process large ECG data. These devices are battery-driven and have low processor and memory capacities. In this work, a novel, naïve yet effective algorithm is presented, which reduces the size of ECG signals in real-time and can be implemented within the constraints of ECG wearable sensor. Our reduced ECG signals reflect the raw ECG signals. In addition, our investigation also found a link between the widow size that used to reduce ECG signal and the quality of the reduced ECG signal. We infer that most intervals including the QT interval must be accurately recognized from reduced ECG data with a window size of 5 . The RR intervals data recognized from the reduced data have $98 \%$ similarity to the
RR data recognized from the original ECG data up to a window size start from 2 to 10 . The proposed technique outperformed other existing real-time reduction and compression ECG data techniques with compression ratio of 5:1 and with low energy consumption. However, when the window size increased $\mathrm{P}$ and $\mathrm{T}$ points were missed leading to failure to detect ST and PR intervals.

\section{ACKNOWLEDGMENT}

Ragheed expresses his special thanks to the Ministry of Higher Education \& Scientific Research and The University of Technology in Iraq for supporting this work.

\section{REFERENCES}

[1] R.-G. Lee, K.-C. Chen, C.-C. Hsiao, and C.-L. Tseng, "A mobile care system with alert mechanism," IEEE Transactions on Information Technology in Biomedicine, vol. 11, pp. 507-517, 2007.

[2] C. G. Scully, J. Lee, J. Meyer, A. M. Gorbach, D. GranquistFraser, Y. Mendelson, et al., "Physiological parameter monitoring from optical recordings with a mobile phone," IEEE Transactions on Biomedical Engineering, vol. 59, pp. 303-306, 2012.

[3] J. J. Oresko II, "Portable heart attack warning system by monitoring the ST segment via smartphone electrocardiogram processing," Citeseer, 2010.

[4] M. Elgendi, B. Eskofier, S. Dokos, and D. Abbott, "Revisiting QRS detection methodologies for portable, wearable, batteryoperated, and wireless ECG systems," PloS one, vol. 9, p. e84018, 2014.

[5] S. Padhy, L. Sharma, and S. Dandapat, "Multilead ECG data compression using SVD in multiresolution domain," Biomedical Signal Processing and Control, vol. 23, pp. 1018,2016

[6] R. Francescon, M. Hooshmand, M. Gadaleta, E. Grisan, S. K. Yoon, and M. Rossi, "Toward lightweight biometric signal processing for wearable devices," in 2015 37th Annual International Conference of the IEEE Engineering in Medicine and Biology Society (EMBC), 2015, pp. 4190-4193.

[7] H. Mamaghanian, N. Khaled, D. Atienza, and P. Vandergheynst, "Compressed sensing for real-time energyefficient ECG compression on wireless body sensor nodes," IEEE Transactions on Biomedical Engineering, vol. 58, pp. 2456-2466, 2011.

[8] G. B. Moody, K. Soroushian, and R. G. Mark, "ECG data compression for tapeless ambulatory monitors," Computers in Cardiology, vol. 14, pp. 467-470, 1987.

[9] S. Lee, J. Kim, and M. Lee, "A real-time ECG data compression and transmission algorithm for an e-health device," IEEE Transactions on Biomedical Engineering, vol. 58, pp. 2448-2455, 2011.

[10] Y. Zigel, A. Cohen, and A. Katz, "The weighted diagnostic distortion (WDD) measure for ECG signal compression," IEEE Transactions on Biomedical Engineering, vol. 47, pp. 1422-1430, 2000.

[11] K. Ranjeet, A. Kumar, and R. K. Pandey, "ECG signal compression using different techniques," in Advances in Computing, Communication and Control, ed: Springer, 2011, pp. 231-241. 
[12] K. Ranjeet, A. Kumar, and R. K. Pandey, "An efficient compression system for ECG signal using QRS periods and CAB technique based on 2D DWT and Huffman coding," in Control, Automation, Robotics and Embedded Systems (CARE), 2013 International Conference on, 2013, pp. 1-6.

[13] S. M. Jalaleddine, C. G. Hutchens, R. D. Strattan, and W. A. Coberly, "ECG data compression techniques-a unified approach," IEEE transactions on Biomedical Engineering, vol. 37, pp. 329-343, 1990.

[14] M. Abo-Zahhad, A. F. Al-Ajlouni, S. M. Ahmed, and R. J. Schilling, "A new algorithm for the compression of ECG signals based on mother wavelet parameterization and bestthreshold levels selection," Digital Signal Processing, vol. 23, pp. 1002-1011, 2013.

[15] A. Ibaida, D. Al-Shammary, and I. Khalil, "Cloud enabled fractal based ECG compression in wireless body sensor networks," Future Generation Computer Systems, vol. 35, pp. 91-101, 2014.

[16] R. J. Hyndman and G. Athanasopoulos, Forecasting: principles and practice: OTexts, 2014.

[17] J. Cox, F. Nolle, H. Fozzard, and G. Oliver, "AZTEC, a preprocessing program for real-time ECG rhythm analysis," IEEE Transactions on Biomedical Engineering, vol. 2, pp. 128-129, 1968.

[18] W. C. Mueller, "Arrhythmia detection program for an ambulatory ECG monitor," Biomedical sciences instrumentation, vol. 14, pp. 81-85, 1977.
[19] R. N. Horspool and W. J. Windels, "An LZ approach to ECG compression," in CBMS, 1994, pp. 71-76.

[20] J. P. Abenstein and W. J. Tompkins, "A new data-reduction algorithm for real-time ECG analysis," IEEE Transactions on Biomedical Engineering, pp. 43-48, 1982.

[21] B. Singh, A. Kaur, and J. Singh, "A Review of ECG Data Compression Techniques," International Journal of Computer Applications, vol. 116, 2015.

[22] S. Tai, "Slope-A real-time ECG data compressor," Medical and biological engineering and computing, vol. 29, pp. 175179, 1991.

[23] W.-C. Fang, H.-C. Huang, and S.-Y. Tseng, "Design of heart rate variability processor for portable 3-lead ECG monitoring system-on-chip," Expert Systems with Applications, vol. 40, pp. 1491-1504, 2013.

[24] T. Jeon, B. Kim, M. Jeon, and B.-G. Lee, "Implementation of a portable device for real-time ECG signal analysis," Biomedical engineering online, vol. 13, p. 1, 2014.

[25] Shimmer ECG sensor. (2016, March 12). [Online]. Available: http://www.shimmersensing.com

[26] R. Mark and G. Moody, "Mit-bih arrhythmia database 1997," URL http://ecg. mit. edu/dbinfo. html, 1997.

[27] I. Silva and G. B. Moody, "An open-source toolbox for analysing and processing physionet databases in matlab and octave," Journal of open research software, vol. 2, 2014. 